\title{
Renal cell carcinoma primary cultures maintain genomic and phenotypic profile of parental tumor tissues
}

\author{
Ingrid Cifola ${ }^{1 \dagger}$, Cristina Bianchi ${ }^{2 \dagger}$, Eleonora Mangano ${ }^{1}$, Silvia Bombelli ${ }^{2}$, Fabio Frascati ${ }^{1}$, Ester Fasoli ${ }^{3}$, \\ Stefano Ferrero ${ }^{3}$, Vitalba Di Stefano ${ }^{2}$, Maria A Zipeto ${ }^{2}$, Fulvio Magni ${ }^{2}$, Stefano Signorini ${ }^{4}$, Cristina Battaglia ${ }^{{ }^{*}}$ and \\ Roberto A Perego ${ }^{2^{*}}$
}

\begin{abstract}
Background: Clear cell renal cell carcinoma (ccRCC) is characterized by recurrent copy number alterations (CNAs) and loss of heterozygosity $(\mathrm{LOH})$, which may have potential diagnostic and prognostic applications. Here, we explored whether $\mathrm{CCRCC}$ primary cultures, established from surgical tumor specimens, maintain the DNA profile of parental tumor tissues allowing a more confident CNAs and LOH discrimination with respect to the original tissues.

Methods: We established a collection of 9 phenotypically well-characterized ccRCC primary cell cultures. Using the Affymetrix SNP array technology, we performed the genome-wide copy number (CN) profiling of both cultures and corresponding tumor tissues. Global concordance for each culture/tissue pair was assayed evaluating the correlations between whole-genome CN profiles and SNP allelic calls. CN analysis was performed using the two CNAG v3.0 and Partek software, and comparing results returned by two different algorithms (Hidden Markov Model and Genomic Segmentation).

Results: A very good overlap between the CNAs of each culture and corresponding tissue was observed. The finding, reinforced by high whole-genome CN correlations and SNP call concordances, provided evidence that each culture was derived from its corresponding tissue and maintained the genomic alterations of parental tumor. In addition, primary culture DNA profile remained stable for at least 3 weeks, till to third passage. These cultures showed a greater cell homogeneity and enrichment in tumor component than original tissues, thus enabling a better discrimination of CNAs and LOH. Especially for hemizygous deletions, primary cultures presented more evident $\mathrm{CN}$ losses, typically accompanied by $\mathrm{LOH}$; differently, in original tissues the intensity of these deletions was weaken by normal cell contamination and LOH calls were missed.

Conclusions: $c$ RCC primary cultures are a reliable in vitro model, well-reproducing original tumor genetics and phenotype, potentially useful for future functional approaches aimed to study genes or pathways involved in ccRCC etiopathogenesis and to identify novel clinical markers or therapeutic targets. Moreover, SNP array technology proved to be a powerful tool to better define the cell composition and homogeneity of RCC primary cultures.
\end{abstract}

\footnotetext{
*Correspondence: cristina.battaglia@unimi.it; roberto.perego@unimib.it

+ Contributed equally

${ }^{2}$ Department of Experimental Medicine, University of Milano-Bicocca, via

Cadore 48, 20052 Monza, Milan, Italy

${ }^{5}$ Dipartimento di Scienze e Tecnologie Biomediche, Università degli Studi di

Milano, via Fratelli Cervi 93, 20090 Segrate, Milan, Italy

Full list of author information is available at the end of the article
} 


\section{Background}

The clear cell subtype of renal cell carcinoma (ccRCC) accounts for $85 \%$ of all RCCs and occurs as familial or, more often, sporadic forms. It is characterized by recurrent genetic anomalies, like copy number alterations (CNAs) and loss of heterozygosity (LOH), that involve specific chromosomes (chrs) and result in deletions with $\mathrm{LOH}$ on chrs $3 \mathrm{p}$ (often involving the von Hippel Lindau (VHL) locus in 3p26-p25), 6q, 8p, 9p and 14q, and duplications of chrs $5 q$ and 7 [1-3]. Evidences suggest that this peculiar pattern of genomic instability represents a tumor-specific molecular fingerprint useful for diagnostic and prognostic applications [3-5]. However, more work still needs to completely clarify the complex molecular pathogenesis of ccRCC. Although the involvement of the $V H L$ tumor suppressor gene has been demonstrated in all familial and in $80-90 \%$ sporadic ccRCCs, the remaining 10-20\% harbors wild-type $V H L$ $[6,7]$, suggesting that, despite their identical histological phenotype, these tumors have an intrinsic molecular heterogeneity that still needs to be unraveled $[6,8]$. The recognition of this molecular heterogeneity might improve the selection of patients for targeted therapies and allow the identification of specific oncogenes and tumor suppressor genes to be used as novel clinical markers or therapeutic targets.

The current availability of high-throughput platforms to assess molecular changes at genome-wide level might provide an opportunity to achieve this goal. Presently a comprehensive and detailed genomic profiling of DNA alterations is possible by using the single nucleotide polymorphism (SNP) array technology. Unlike CGH technique, the SNP array platform allows the simultaneous analysis of both chromosomal and allelic imbalances [9] and the distinction between $\mathrm{LOH}$ associated with $\mathrm{CN}$ changes (such as hemizygous deletions) and $\mathrm{CN}$ neutral status (often termed as uniparental disomy) [10]. In addition, this technology is able to provide information about the fraction of normal and tumor cells present in the tumor tissue samples [11]. This allows a more complete definition of the complex genetic rearrangements associated with cancer pathologies. Moreover, since the regions of LOH accompanied by deletion (representing the second hit of Knudson's hypothesis) are of particular interest because they may contain genes involved in tumor etiology, the assessment of deletion/LOH areas represents a useful approach to identify regions potentially harboring novel tumor suppressor genes [12]. Accordingly, in a previous study, we used the SNP array technology to characterize the whole-genome DNA profile of a collection of ccRCC tissue samples to find novel chromosomal regions and genes potentially interesting as candidate tumor markers [13].
However, in ccRCC specimens, as in most solid tumor tissues, the molecular analyses may be affected by tissue heterogeneity due to the presence of necrotic areas and non-tumor cells, such as tumor-infiltrating leukocytes, endothelial cells and fibroblasts [14]. With the purpose to increase the quality of data by minimizing this "background noise", several different technical approaches have been explored [15]. Tissue heterogeneity must be considered also when evaluating which are the most appropriate computational tools to process and analyze array-based $\mathrm{CN}$ data $[11,16]$.

Thus, to overcome the problem of tissue heterogeneity and to prospectively perform in vitro functional studies aimed to better understand ccRCC molecular pathogenesis, it is necessary to have a viable and more homogeneous cell material retaining the phenotypic and genomic profile of original tissue. A possible strategy to face these requirements is to adapt fresh ccRCC tissue specimens to grow in vitro as primary cell cultures, which provide a good quality, homogeneous and wellcharacterized cellular material, enriched with tumor cell component [17] and retaining at the first passages the phenotypic and proteomic profile of the corresponding tissues from which they derive $[15,18,19]$. Hence, primary cultures represent a better in vitro tumor model than stable cell lines, that can be even very different from the original tissues and thus not at all representative [6]. Anyway, the reliability of data obtained from primary cultures strictly depends on their careful cytological characterization, especially regarding possible cell contaminations that might influence data interpretation with misleading effects.

In this study, we investigated whether ccRCC primary cultures, established from surgical tumor specimens and phenotypically well-characterized, maintain the DNA profile of parental tumor tissues and allow a better discrimination of CNAs and $\mathrm{LOH}$ with respect to original tissues. Till now, the comparison of DNA profile between tumor primary cultures and parental tissues has been reported, with discordant results, in melanoma [20], neuroblastoma [21] and glioblastoma [22], using either SNP array or array-CGH techniques, and in RCC [23] only using traditional CGH on metaphase spreads and short-term primary cultures not extensively characterized.

\section{Methods \\ CCRCC primary culture preparation and immunophenotypic characterization}

Nine ccRCC primary cultures were established from corresponding surgical tissue specimens of ccRCC cases (Table 1). Patients were enrolled in the research project at Policlinico Hospital (University of Milan, Italy) and provided informed consent for the research use of 
Table 1 Clinical data of the nine clear cell RCC cases from which primary cultures have been prepared

\begin{tabular}{ccccc}
\hline Case & Gender & $\begin{array}{c}\text { Age } \\
\text { (years) }\end{array}$ & $\begin{array}{c}\text { Tumor size } \\
\text { (cm) }\end{array}$ & $\begin{array}{c}\text { Tumor stage (pT)/ } \\
\text { Grade }\end{array}$ \\
\hline 50PC & $\mathrm{M}$ & 51 & 5.7 & $\mathrm{pT} 1 / \mathrm{G} 2$ \\
$59 \mathrm{RG}$ & $\mathrm{M}$ & 72 & 5 & $\mathrm{pT} 1 / \mathrm{G} 2$ \\
$60 \mathrm{CC}$ & $\mathrm{M}$ & 78 & 11.5 & $\mathrm{pT} / \mathrm{G} 3$ \\
$61 \mathrm{FG}$ & $\mathrm{M}$ & 56 & 5 & $\mathrm{pT} 1 / \mathrm{G} 2$ \\
$66 \mathrm{SML}$ & $\mathrm{F}$ & 48 & 9 & $\mathrm{pT} / \mathrm{G} 2$ \\
70LS & $\mathrm{M}$ & 71 & 4.4 & $\mathrm{pT} 1 / \mathrm{G} 2$ \\
73PG & $\mathrm{F}$ & 75 & 2.5 & $\mathrm{pT} 1 / \mathrm{G} 3$ \\
80MLa & $\mathrm{F}$ & 78 & 6.2 & $\mathrm{pT} / \mathrm{G} 2$ \\
81BPG & $\mathrm{M}$ & 69 & 3.3 & $\mathrm{pT} 1 / \mathrm{G} 2$ \\
\hline
\end{tabular}

leftover material. Study protocol and procedures were approved by the local ethic committee. All RCC cases were diagnosed as "clear cell" subtype independently by two pathologists with expertise in kidney cancer. Prior to surgery, a whole blood sample was collected for each case and stored at $-20^{\circ} \mathrm{C}$. Immediately after surgical removal of the kidney, sections of fresh tissue samples enriched in tumor component by needle dissection were both stored at $-80^{\circ} \mathrm{C}$ and collected in cold DMEM medium supplemented with $10 \%$ fetal calf serum (FCS), $1 \%$ penicillin/streptomycin, $1 \%$ amphotericin and $1 \%$ glutamine (Culture Medium) and kept at $4{ }^{\circ} \mathrm{C}$ until processing (within $24 \mathrm{hrs}$ ). After removal of adipose and necrotic areas, tumor tissues were mechanically minced in 1 $\mathrm{mm}^{3}$-fragments and digested with $25 \mathrm{mg} / \mathrm{ml}$ collagenase type IV (Sigma-Aldrich, St Louis, MO, USA) in DMEM medium for 2 hrs at $37^{\circ} \mathrm{C}$, vigorously vortexing every 15 minutes. Then, samples were washed three times in PBS at $4^{\circ} \mathrm{C}$ and plated in $60 \mathrm{~mm}$-Petri dishes or on glass cover slips in the Culture Medium and incubated at $37^{\circ}$ $\mathrm{C}$ in $5 \% \mathrm{CO}_{2}$. Medium was changed twice weekly and cells were 1:3 splitted when reaching $90 \%$ confluence. Cell morphology was observed in contrast phase, at 100× magnification, by Olympus CK40 inverted microscope (Olympus Corporation, Tokyo, Japan).

For immunofluorescence microscopy, ccRCC cells grown on glass cover slips were fixed for $30 \mathrm{~min}$ in $4 \%$ paraformaldehyde $\mathrm{PBS}$ buffer, $\mathrm{pH} 7.2$, at $37^{\circ} \mathrm{C}$, rinsed with PBS, pre-incubated for 15 min in GDB buffer $(0.02$ $\mathrm{mol} / \mathrm{l}$ sodium phosphate buffer, $\mathrm{pH} 7.4$, with $0.45 \mathrm{~mol} / \mathrm{l}$ $\mathrm{NaCl}$ and $0.5 \% \mathrm{BSA}$ ) containing $0.3 \%$ Triton X-100, and incubated with mouse monoclonal antibodies (mAbs) against pan-cytokeratin (clone MNF-116, Dako, Glostrup, Denmark; dilution 1:200), vimentin (clone V9, Dako; dilution 1:200), carbonic anhydrase IX (CA9) (clone M75, dilution 1:50; a gift from Prof. Pastorekova, Institute of Virology, Slovak Academy of Sciences, Bratislava, Slovak Republic) and FITC-conjugated mAb against CD13 (clone CBL 169F, Chemicon, Billerica,
MA, USA; dilution 1:25) for 2 hrs at room temperature. After washing in PBS, cover slips were incubated for 1 hr with goat anti-mouse Alexa-Fluor488-conjugated IgG secondary antibody (Molecular Probes, Invitrogen Life Technologies, Carlsbad, CA, USA; dilution 1:100). Nuclear counterstaining was performed by incubation for 5 min with $1 \mu \mathrm{M}$ DAPI (Sigma-Aldrich) in PBS buffer. Immunofluorescence micrographs were obtained using a Zeiss Axiovert 200 inverted microscope (Zeiss Inc., Oberkochen, Germany), at 400× magnification, equipped with a CoolSNAP HQ camera driven by Metamorph software. For flow cytometry analysis, cells at the first confluence ( 1$)$ were detached from plates with $0.25 \%$ trypsin-EDTA (Sigma-Aldrich), rinsed and incubated for 15 min in PBS with 5\% FCS. For CD13 and CA9 staining, cells were incubated for $15 \mathrm{~min}$ at room temperature with the specific mouse mAbs. Cytokeratin and vimentin staining was performed using the specific mouse mAbs after permeabilization with IntraStain solution (Dako). Cells were then incubated with goat antimouse Alexa-Fluor488-conjugated IgG secondary antibody (Molecular Probes, Invitrogen Life Technologies) for $30 \mathrm{~min}$ at $4^{\circ} \mathrm{C}$ and counted by the FACSCalibur flow cytometer and CellQuest software (BD Biosciences) until 30,000 events were acquired.

\section{Western Blot analysis}

For each case, cells plated in a $6 \mathrm{~mm}$-Petri dish were lysated when at the first confluence ( 11$)$, and the extracted proteins quantified by the Bio-Rad microassay (BioRad, Hercules, CA, USA), as previously described [18]. $30 \mu \mathrm{g}$ proteins were separated on NuPage 4-12\% Bis-Tris pre-cast gels (Invitrogen Life Technologies) and blotted onto nitrocellulose membranes probed in 10 $\mathrm{mM}$ Tris- $\mathrm{HCl}, \mathrm{pH} 8$, and $3 \% \mathrm{BSA}$, with mouse $\mathrm{mAb}$ against CA9 (clone M75, dilution 1:50). Detection was performed using a secondary antibody coupled with horseradish peroxidase for $1 \mathrm{hr}$ at room temperature and the SuperSignal West Pico detection system (Pierce, Rockford, IL, USA).

\section{DNA extraction and target preparation for Affymetrix SNP Arrays}

The genomic studies were performed on all ccRCC primary cultures at first confluence (p1); in addition, for 80MLa and 81BPG cases also cultures at second confluence (p2) or at second (p2) and third (p3) confluences were characterized. Genomic DNA was extracted from primary cell cultures by QIAmp DNA Mini kit (Qiagen, Hilden, Germany), according to manufacturer's cultured cell protocol. DNA from tumor tissues and autologous whole blood samples, used as normal control, was extracted using a standard proteinase $\mathrm{K}$ cell lysis and phenol-chloroform procedure. Samples were quantified 
by ND-1000 spectrophotometer (NanoDrop Technologies, Wilmington, DE, USA) and checked by electrophoresis on $0.8 \%$ agarose gel. Starting from $250 \mathrm{ng}$, DNA samples were processed using the GeneChip ${ }^{B}$ Human Mapping 50K Hind Assay kit (Affymetrix, Santa Clara, CA, USA), according to manufacturer's protocol, and hybridized onto GeneChip ${ }^{\circledR}$ Human Mapping 50K Hind Arrays. Intensity signals were acquired by Affymetrix GeneChip ${ }^{\circledR}$ Scanner $30007 \mathrm{G}$ and quantified by GTYPE v4.1 software (Affymetrix), using the BRLMM algorithm to assign SNP calls and to generate CHP files. A SNP call rate greater than $95 \%$ was considered as "good quality" threshold.

\section{Genome-wide copy number and LOH profiling in cCRCC primary cultures and tumor tissues}

To assess copy number alterations (CNAs) and loss of heterozygosity (LOH), two different software CNAG (version 3.0) and Partek Genomics Suite (version 6.5) were applied. CNAG is a well known software commonly used to analyze SNP array derived-CN data in tumor samples [24]. The AsCNAR (allele-specific copynumber analysis using anonymous reference) algorithm was applied to perform a "self-reference paired analysis", by comparing each culture and corresponding tumor tissue to the matched blood sample. All resulting profiles (global $\log$ ratio $\mathrm{CN}$ profiles; allele log ratio $\mathrm{CN}$ profiles; Hidden Markov Model (HMM)-inferred CN state; HMM-inferred LOH) were visually inspected to identify regions affected by aberrations. To increase data reliability, we chose to include only CNAs longer than $2 \mathrm{Mb}$ (resolution limit of 50K SNP array platform). CNAG software was also used to obtain a genome-wide map of $\mathrm{LOH}$ events occurring in each culture or tumor tissue with respect to matched blood. Only the $\mathrm{LOH}$ events defined as statistically significant by CNAG (with LOH likelihood higher than 30, as default threshold, and thus visualized in the HMM-LOH track) and covering regions longer than $2 \mathrm{Mb}$ were considered.

The same paired analysis was performed using Partek Genomics Suite software (Partek Inc., St Louis, MO, USA), starting from CEL intensity files and applying the two alternative algorithms Hidden Markov Model (HMM, the same used by CNAG) and Genomic Segmentation (GS). For HMM analysis, default parameters were adopted; for GS analysis, the default window of 10 contiguous SNPs was maintained, Signal to noise ratio at 0.5 and p-value at 0.001 were also adopted.

To estimate the global concordance between the genomic profile of each primary culture and its parental tissue, a paired whole-genome $\mathrm{CN}$ correlation was calculated, starting from the CNAG "HMM-CN state" data (SNP copy number status inferred by HMM), and applying Spearman's regression method. SNP call concordance index and Spearman's correlation between the CNAG SNP allelic calls of two matched samples were also calculated. For SNP call concordance, a contingency table of the counts for each combination of the four genotype categories (AA, AB, BB, NoCall) for culture (in row) and matched tissue (in column) was accomplished. The concordance index was calculated as a ratio between the sum of principal diagonal counts and 56,859 (total number of real informative SNPs on $50 \mathrm{~K}$ Hind arrays), multiplied by 100 . A SNP call concordance higher than $60 \%$ for two related samples was adopted as threshold. All statistical analysis were performed in R environment.

\section{Results}

\section{Phenotypic characterization of ccRCC primary cultures}

The nine ccRCC primary cultures established from surgical tissue specimens reached the first confluence ( $\mathrm{p} 1$ ) in $8.0 \pm 2.1$ days. They grew well till reaching the fourth confluence, and then began to slow down their growth rate. In particular, 80MLa and 81BPG cultures reached the second confluence (p2) after 13 days, and 81BPG culture reached the third confluence (p3) after 20 days. Cells exhibited heterogeneous epithelioid morphology, were able to form foci and presented cytoplasmic vacuoles frequent in clear cell RCC subtype during in vitro growth (Figure 1a). Cytoplasmic staining specific for the epithelial cytokeratin and for vimentin, a mesenchymal marker expressed also in the epithelial RCC cells, both in vivo and in vitro $[18,25]$, and specific expression of the proximal tubular marker CD13 were observed in more than $90 \%$ of cells, as assessed by immunofluorescence and FACS analysis, according to the proximal tubular origin of ccRCC (Figure 1b). Immunofluorescence and FACS analysis were performed also with the monoclonal antibody against the transmembrane carbonic anhydrase IX (CA9) protein, one of the most used biomarkers for ccRCC $[26,27]$. Our ccRCC primary cultures showed the typical pattern of membrane fluorescence and more than $60 \%$ of cells were positive for CA9 (Figure 1b). Moreover, protein lysates analyzed by Western Blot with anti-CA9 antibody showed the expected doublet at about $55 \mathrm{kDa}$ in all samples, except for 73PG culture (Figure 1c). On the whole, these results confirmed the neoplastic phenotype of our tubular primary cultures.

\section{Genome-wide assessment of CNAs and LOH in CCRCC primary cultures}

The Affymetrix 50K SNP Array platform was used to perform the whole-genome SNP profiling of 12 samples from ccRCC primary cultures, nine at the first confluence (p1), two at the second confluence (p2), and one at the third confluence (p3). In addition, 9 samples from 
a

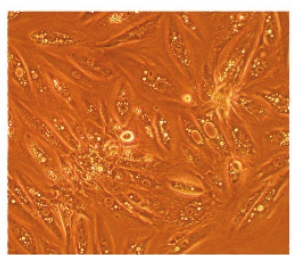

b
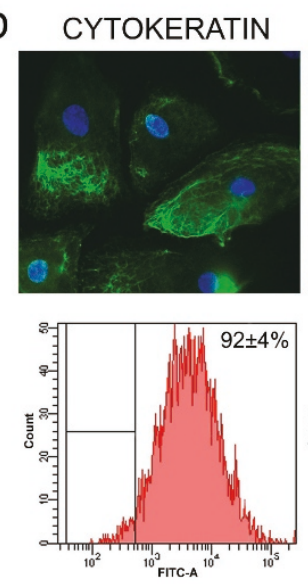

C

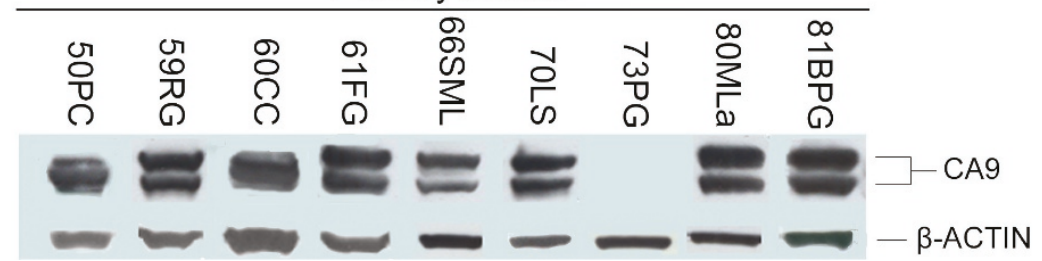

VIMENTIN
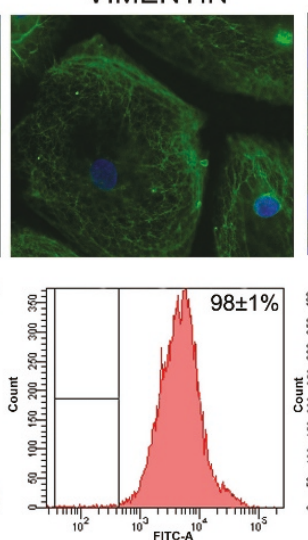

CD13
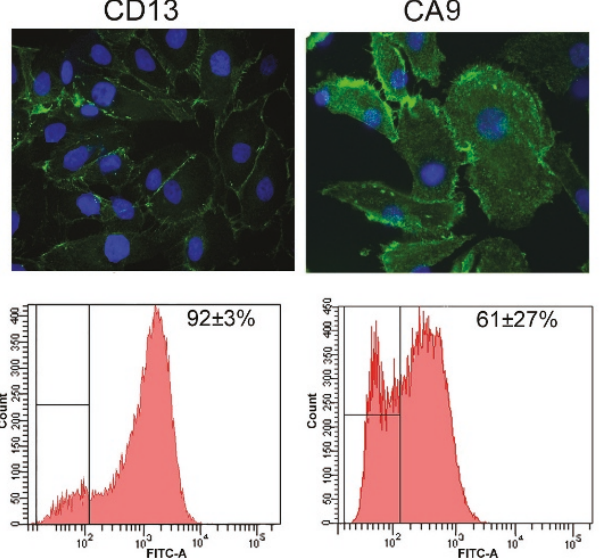

Figure 1 Phenotypic characterization of ccRCC primary cultures. (a) Representative cellular morphology during in vitro growth. 100x
magnification. (b) Representative micrographs of immunofluorescence staining (top) and FACS analysis (bottom) of pan-cytokeratin, vimentin, CD13 and CA9. DAPI counterstains nuclei in blue. 400x magnification. The positivity percentages for the different markers are reported in the FACS analysis as mean value ( \pm SD) of the nine cultures. (c) Western Blot analysis of CA9 in all ccRCC primary cultures. B-actin was used as internal control.

the corresponding original tumor tissues and 9 autologous blood samples were analyzed. We obtained an average SNP call rate equal to $98.54 \%$, ranging from $95.79 \%$ to $99.67 \%$, and all arrays were included in the analyses. Using CNAG v3.0 software, we performed the genome-wide profiling of CNAs in each tumor primary culture at p1. Globally, the typical "clear cell RCC" genomic signature was confirmed, including deletions on chr $3 p$ and amplifications on chrs $5 q$ and 7 (see Additional File 1), the same signature that we previously described in another set of ccRCC tissue samples [13].

\section{Genome-wide comparison of DNA profile of primary cultures and original tumor tissues}

Our main purpose was to assess if ccRCC primary cultures reflected the genomic profile of tumor tissues from which they derived. First of all, for each tumor primary culture/tissue pair, we calculated the global correlation coefficient (by Spearman regression) between their whole-genome CN profiles. Starting from the HMM-CN state data generated by CNAG, we obtained a mean $\mathrm{CN}$ correlation value equal to 0.73 (range 0.30-0.99) (Table 2). This wide range of variation was due essentially to 66SML sample, which presented the lowest CN correlation coefficient (Spearman
0.30). On the other hand, both the concordance index and the Spearman's correlation calculated on CNAG SNP allelic calls gave very high values for all cases (SNP call concordance indexes from $94 \%$ to $98 \%$ and Spearman's coefficients from 0.87 to 0.96 ), thus indicating a strong correlation between cultures and corresponding tissues at SNP genotype level, also for the 66SML case (Table 2).

Table 2 Estimations of the global correlation between the genomic profile of each primary culture and corresponding parental tissue

\begin{tabular}{cccc}
\hline Sample & $\begin{array}{c}\text { Spearman's } \\
\text { correlation on HMM- } \\
\text { CN state }\end{array}$ & $\begin{array}{c}\text { SNP call } \\
\text { concordance } \\
\text { index }\end{array}$ & $\begin{array}{c}\text { Spearman's } \\
\text { correlation on } \\
\text { SNP calls }\end{array}$ \\
\hline 50PC & 0.66 & $98 \%$ & 0.96 \\
59RG & 0.54 & $98 \%$ & 0.96 \\
60CC & 0.94 & $94 \%$ & 0.87 \\
61FG & 0.97 & $98 \%$ & 0.96 \\
66SML & 0.30 & $96 \%$ & 0.94 \\
70LS & 0.99 & $98 \%$ & 0.96 \\
73PG & 0.68 & $97 \%$ & 0.95 \\
80MLa & 0.60 & $97 \%$ & 0.93 \\
81BPG & 0.90 & $95 \%$ & 0.92 \\
\hline
\end{tabular}


Looking at the individual $\mathrm{CN}$ profiles produced by CNAG for each sample, 7 out of 9 primary cultures exactly maintained all the DNA alterations presented by the corresponding tumor tissues, or retained the normal $\mathrm{CN}$ profile as observed in the original tissue (73PG case) (Figure 2). The remaining two cultures presented the DNA profile of parental tissues except for one CNA: 59RG culture did not show the chr 16p amplification and 66SML culture did not show the chr $1 \mathrm{p}$ deletion observed in the corresponding tissues. Moreover, four primary cultures presented additional CNAs on one or two chromosomes, not found in original tissues: 50PC and $60 \mathrm{CC}$ cultures had an additional amplification on chr 22q, 59RG showed amplification of chrs 2 and 7, whereas 81BPG showed deletion with LOH on chrs 8p and 14q (Figure 2).

Concerning LOH profile, 60CC culture exactly maintained all the allelic imbalances, with corresponding $\mathrm{CN}$ status, found in the original tumor tissue, while 50PC, 61FG and 73PG cultures confirmed the absence of LOH observed in parental tissues (Figure 2). The remaining five primary cultures presented a total of $11 \mathrm{LOH}$ regions that were not detected by CNAG in original tissues because not reaching the $\mathrm{LOH}$ likelihood threshold to be classified as statistically significant by the software and thus visualized in the HMM-LOH track. Viceversa, we did not found $\mathrm{LOH}$ events occurring in tumor tissues and not confirmed in corresponding cultures.

Additionally, we performed the whole-genome DNA profiling of $80 \mathrm{MLa}$ culture also at second (p2) confluence (see Additional File 2) and of 81BPG culture at second (p2) and third (p3) confluences (Figure 3). In both cases, all the CNAs and $\mathrm{LOH}$ observed at $\mathrm{p} 1$ were exactly maintained at $\mathrm{p} 2$ and $\mathrm{p} 3$, and no other alterations occurred along passages. Even the deletions on chrs $8 \mathrm{p}$ and $14 \mathrm{q}$ found in 81BPG culture at p1, but not in original tissue, were confirmed at $\mathrm{p} 2$ and $\mathrm{p} 3$ (Figure $3)$. On the whole, these results indicated that the genomic profile of ccRCC primary cultures highly reflected that of parental tissues and remained stable during the early passages, thus suggesting that these well-characterized primary cultures may be a good in vitro model of original tumor tissues.

Moreover, when looking more in detail at the $\mathrm{CN}$ profiles calculated by CNAG, we observed that in six primary cultures (59RG, 61FG, 66SML, 70LS, 80MLa, $81 \mathrm{BPG}$ ) the $\mathrm{CN}$ values of aberrant regions were more definite in cultures than in parental tissues and this phenomenon prevalently affected $\mathrm{CN}$ loss events. In the 66SML case, this situation was particularly evident. The primary culture presented 4 wide $\mathrm{CN}$ loss regions on chrs 2q, 3p, 9 and 14q. As shown in Figure 4 for chr 3p

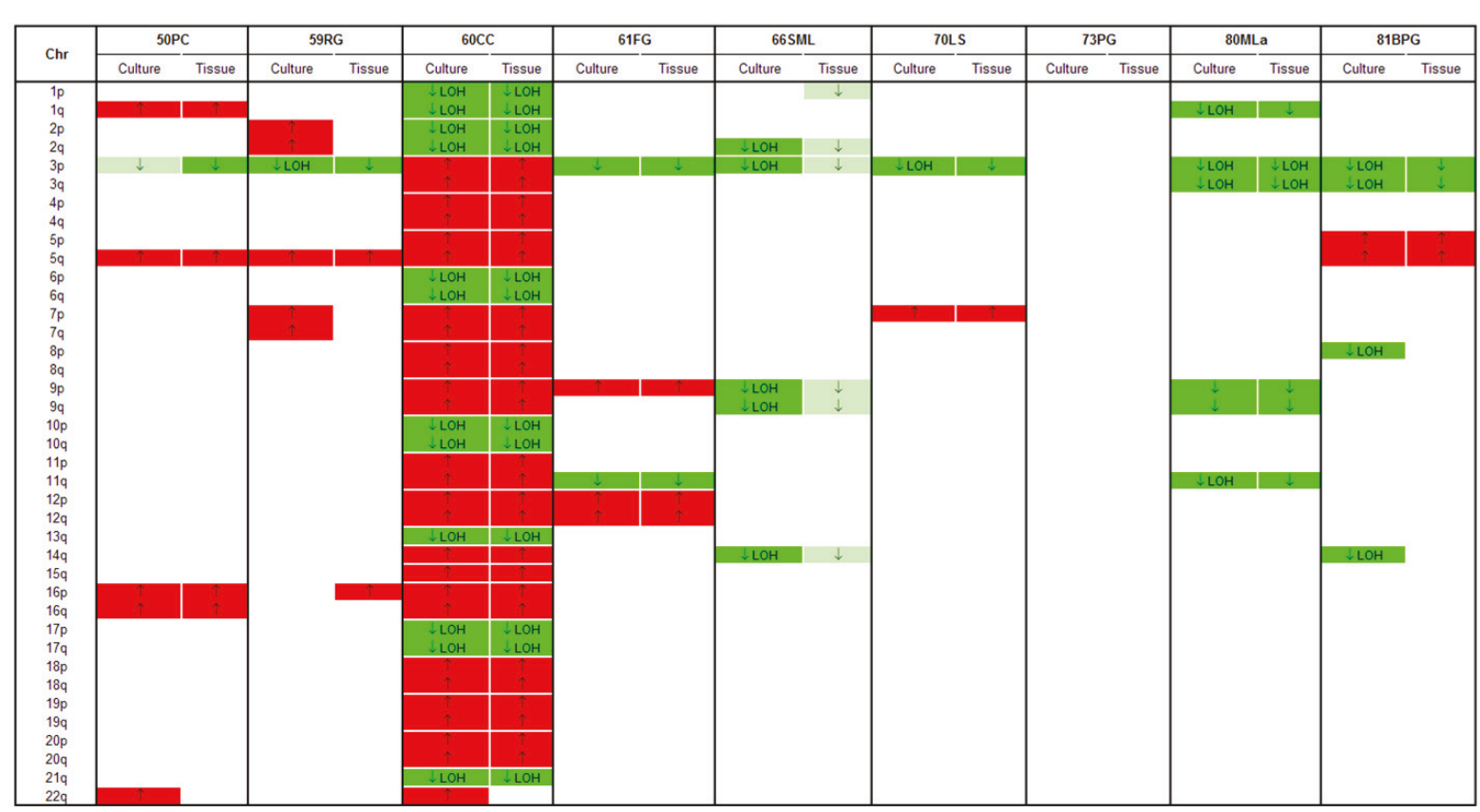

Figure 2 Copy number alterations and LOH events in cCRCC primary cultures and parental tissues, as calculated by CNAG v3.0 software. On each chromosomal arm ( $p$, short arm; $q$, long arm), amplifications ( $\uparrow$ ) and deletions ( $\downarrow$ ) and LOH events are reported for all samples. Color labels distinguish CN alterations (CNAs) detected by CNAG and signed in the color-coded "HMM-CN state" track (red for amplifications and dark green for deletions), and CNAs resulting below threshold to be visualized in the HMM-CN track (light green for deletions). Only LOH events reaching significant likelihood to be signed by CNAG in the HMM-LOH track are reported. 


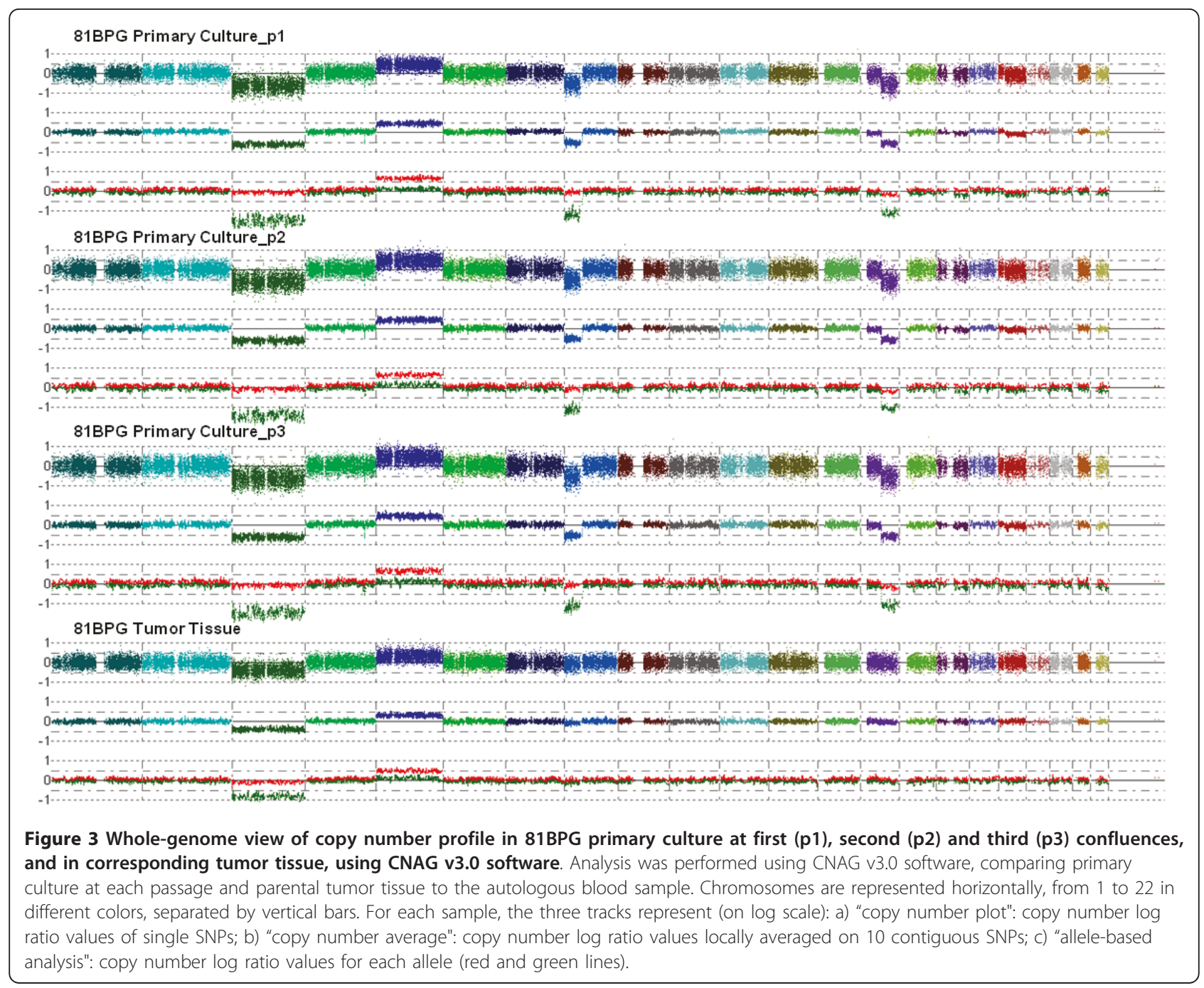

deletion, in primary culture the CNA region was defined by pronounced negative $\mathrm{CN}$ values, easily classified by CNAG software as statistically significant $\mathrm{CN}$ loss. This deletion was present also in the parental tissue and was visible in the $\mathrm{CN}$ profile. However, because of its less pronounced $\mathrm{CN}$ values, it did not reach the software threshold to be signed as statistically significant and did not appear in the HMM-CN state track. In Additional File 3 we reported other $\mathrm{CN}$ loss regions for 66SML case and two other representative samples: calculating the mean $\mathrm{CN}$ values corresponding to each of these deletions, we always observed values more negative in culture than in corresponding parental tissue. This situation reflected also on $\mathrm{LOH}$ profiles. In fact, all the $11 \mathrm{LOH}$ events previously mentioned as detected in primary cultures but not in parental tissues occurred in such deleted regions presenting weak $\mathrm{CN}$ loss values in tissues. As represented in Figure 4 for chr 3p in 66SML, the software still detected the presence of heterozygous
SNP calls in parental tissue (green bars below chromosome ideogram), thus indicating that the hemizygous deletion does not occur in all cells and suggesting the presence of contaminating diploid cells. In all these cases, LOH likelihood consequently decreased below statistical significance threshold and these events were missed to be visualized by CNAG in the HMM-LOH track, as illustrated in Figure 4 (see Additional File 3 for LOH likelihoods of the other deleted regions). Thus, it can be concluded that the increased cell homogeneity of primary cultures, in term of tumor component, in comparison with their parental tumor tissues enabled a better discrimination of CNAs and $\mathrm{LOH}$.

Lastly, we performed the same paired analysis between tumor primary culture and parental tissue using Partek Genomics Suite software, which allows to choose between two alternative $\mathrm{CN}$ algorithms. Globally, using the HMM-based algorithm (the same used by CNAG), Partek returned results overlapping with CNAG analysis. 


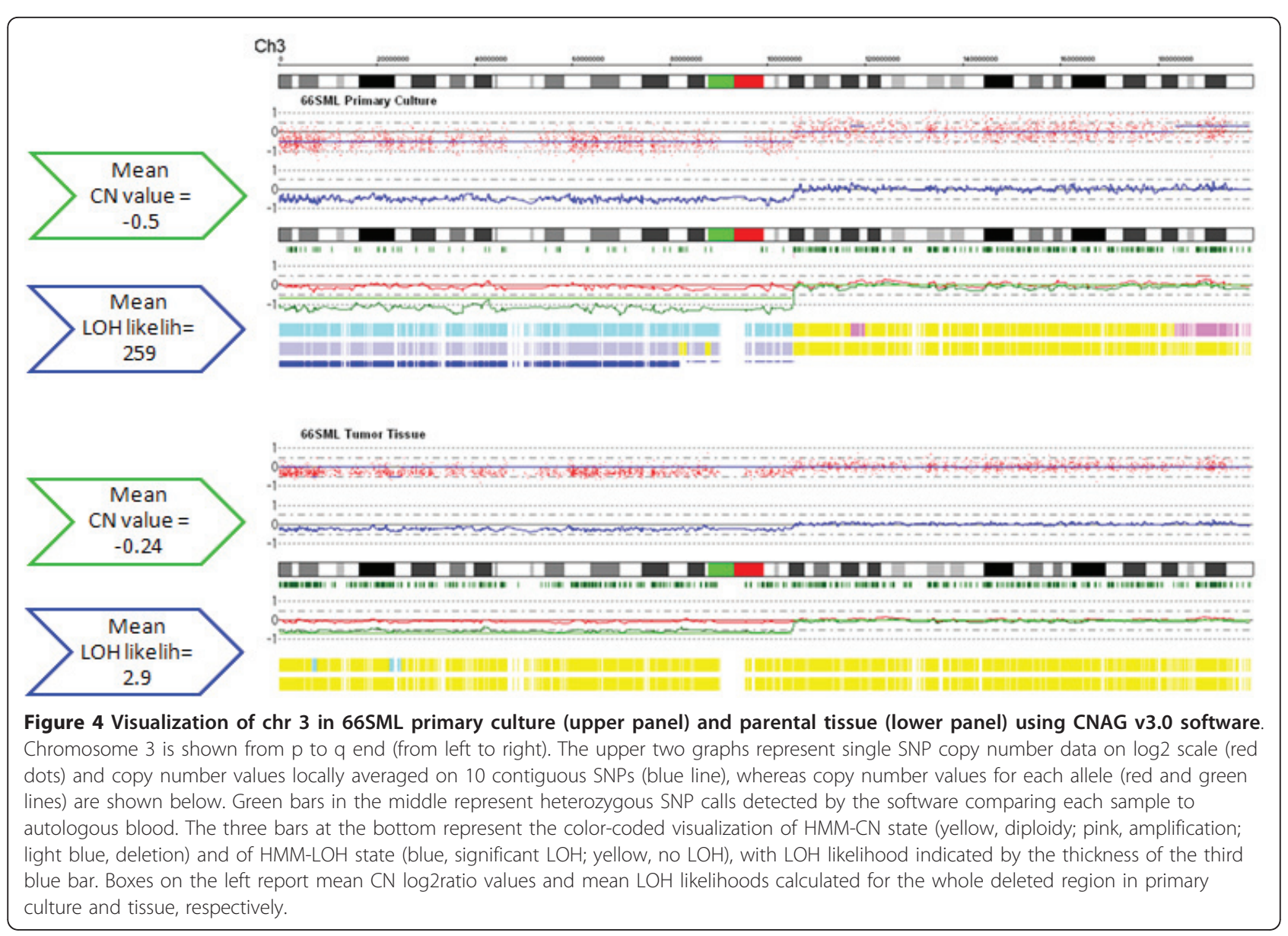

Differently, when applying the GS algorithm, Partek was able to retrieve all those $\mathrm{CN}$ losses missed to be classified as statistically significant in tissue samples by the CNAG HMM algorithm. This is illustrated for 66SML case in Additional File 4. These results confirmed that in tissue samples these deletions were really present even if less visible than in cultures due to sample heterogeneity, and they did not arise de novo in primary cultures.

\section{Discussion}

In this study, we investigated whether the in vitro model of ccRCC primary cell cultures, established from surgical tumor specimens, well reproduces the DNA profile of parental tumor tissues, thus allowing a more confident CNAs and LOH discrimination with respect to the original tissues. Tumor cells from ccRCC surgical specimens may be adapted to in vitro growth with high efficiency, independently from any clinico-pathological characteristic of patients, as we previously confirmed also in a wide series of well-characterized samples $[18,19]$. The growth and survival rate of our ccRCC cultures were in agreement with those reported by other authors [14] and proved that our primary cultures grow for the first four passages without difficulties [18]. The cellular composition of our cultures was very homogeneous: more than $90 \%$ of cultured cells were of proximal tubular origin, with morphological characteristics typical of ccRCC, and more than $60 \%$ of cells were positive for CA9, a biomarker present in almost all ccRCC cases and expressed in most, although not all, of the malignant clear cells of each single positive case $[26,28]$. When evaluated by Western Blot, CA9 expression was quite strong in all samples, except for 73PG culture where it was undetectable. Notably, about this latter culture, we found an identical normal genomic profile in primary culture and parental tissue in terms of both CNAs and LOH (with the resolution power of the $50 \mathrm{~K}$ SNP array platform), although the tumor tissue sample, unlike primary culture, showed a CA9 expression by immunohistochemistry (data not shown). This finding is in agreement with another paper reporting a ccRCC case which expressed CA9 in the surgical tissue sample but not in the corresponding primary culture [15]. Moreover, in our experience with a wide series of samples [19], we observed other few ccRCC cases showing a 
CA9 positivity at tissue level but not in corresponding primary cultures. Excluding tissue sampling mistakes, this behavior might be due to a transitory expression of CA9 during tissue excision. In fact, it is known that surgical conditions, like tissue ischemia after renal artery clamping and subsequent hypoxia, may up-regulate the expression of downstream targets of HIF-1 (hypoxia inducible factor 1), including CA9, that conversely should not be induced under normoxic conditions like those applied for primary cultures [29]. On the other hand, the outgrowth of normal tubular epithelial cells in 73PG culture is unlikely based on the extensive phenotypic characterization we performed on all our cultures [19].

Up to now, karyotypic characterization of RCC primary cultures has been performed only by classical cytogenetic G-banding [30] or $\mathrm{CGH}$ technique [23]. In our knowledge, this is the first study applying the Affymetrix SNP array technology to assess at genome-wide level both CNAs and $\mathrm{LOH}$ in ccRCC primary cultures. Globally, our cultures confirmed the typical ccRCC genomic signature $[13,23,30]$. These cultures typically showed alterations on at most 4 or 5 chromosomes. Only two samples made exception: 73PG culture did not show alterations in both $\mathrm{CN}$ and $\mathrm{LOH}$ profiles, while $60 \mathrm{CC}$ showed CNAs on all chromosomes. In particular, 60CC primary culture, as well as its corresponding tissue, showed an atypical ccRCC genomic profile more similar to that of chromophobe subtype (characterized by wide losses on chrs 1, 2, 6, 10, 13, 17 and 21), notwithstanding its typical "clear cell" histology.

It should also be noted that while 73PG specimen derived from a small in size tumor, suggestive of an early stage of neoplastic progression, 60CC specimen derived from the largest one, probably associated with a more advance stage of tumor progression characterized by an aberrant and "clear cell" atypical genomic profile [2].

Up today, very few genomic comparisons between short-term primary cultures and parental tumor tissues have been performed. In glioblastoma, for example, the genomic profile of primary cultures, assessed by array$\mathrm{CGH}$, resulted considerably different from that of parental tumors, with changes progressively occurring already after 2 weeks of culture, resulting in an inconsistent representation of tumor biology [22]. In melanoma [20] and neuroblastoma [21], instead, using SNP array technology, primary cultures showed to encompass the spectrum of significant alterations present in primary tumors, thus providing a genetically appropriate in vitro model for functional genomics characterizations.

Concerning RCC, up till now the comparisons between primary cultures and tumor tissues mainly regarded phenotypic characterizations and proteomic profiling $[15,18,31]$. A comparison of the genomic profile between primary cultures and parental tissues has been reported only by Sanjmyatav et al. by using traditional CGH [23]. Their DNA profiling showed a poor overlap of CNAs between ccRCC primary cultures and parental tumor tissues. In fact, only 3 out of 8 ccRCC cultures exactly showed the same DNA alterations present in the corresponding tissues. In addition, other 3 cultures did not show any of the CNAs found in the corresponding tissue samples and resulted diploid. Probably, the poor overlap of CNAs obtained by Sanjmyatav et al. [23] was due both to the low sensitivity and resolution level of the technique used (i.e. CGH on metaphase chromosome spreads) and to the not extensive cytological characterization of primary cultures. These findings highlight the importance of a careful phenotypic characterization of primary cultures for a correct interpretation of genomic results.

Globally, these few genomic comparison studies between primary cultures and parental tissues point out that the use of tumor primary cultures as in vitro model for genetic analysis or functional studies must be distinctively evaluated tumor by tumor and that in any case it depends on the level of phenotypic characterization of primary cultures.

Looking at the genome-wide $\mathrm{CN}$ profiles, our results indicated that 7 out of 9 well-characterized primary cultures exactly reproduced the DNA profile of the corresponding tumor tissues. The other two cultures (59RG and 66SML) maintained all but one the CNAs showed by the original tissues. These findings are in agreement with Lin et al. who evidenced that in melanoma some significant alterations present in original tissues (as a deletion on chr 13q) were almost undetectable in cultured cells, despite the landscape of genomic alterations was strikingly similar [20]. However, it must be pointed out that even if primary cultures might have lost some genomic alterations present in original tumor tissues, they probably correspond to some "passenger mutations", that do not confer selective growth advantage to tumor cells and thus might be lost in cultured cells, and not to "driver mutations" [32].

Although the good overlap of $\mathrm{CN}$ profiles between each our primary culture and corresponding tissue, we obtained a CN correlation mean value equal to 0.73 (by Spearman regression method), a value just a little higher than that reported for glioblastoma cultures and tissues (Pearson mean 0.62) [22]. The reason is in the wide range of variance we obtained among our 9 cases (range 0.30-0.99), mainly due to the contribution of one case (66SML) on the mean. Although this variability seems too high for conclusive messages, it must be noted that in our series it essentially depends on a software issue. In fact, we used the CNAG "HMM-CN state" data, that 
is the SNP copy number status inferred by HMM algorithm and visualized in the HMM-CN state track. However, in the presence of a high sample heterogeneity, as in the case of 66SML tissue (illustrated in Figure 4), the HMM-CN state data missed to detect $\mathrm{CN}$ aberrations, even if present, because their $\mathrm{CN}$ values did not reach the software threshold for a statistical significance. For this reason, the 66SML sample had the lowest CN correlation coefficient between culture and tissue (Spearman 0.30), a value that does not really reflect a poor overlap but only a software constraint.

Similarly to those found in neuroblastoma [21], the SNP call concordance indexes and the Spearman's correlation coefficients calculated on CNAG SNP allelic calls indicated a strong correlation at SNP genotype level between our cultures and parental tissues, 66SML sample included, thus confirming that each primary culture really derived from its corresponding tissue.

Notably, in six samples, CNAs were more evident and better discriminated in primary cultures than in corresponding tumor tissues. This phenomenon principally occurred in deleted regions and it is due to the different cellular composition of the two samples and it was also observed in neuroblastoma tissues and derived cell lines [21]. Tumor tissues are heterogeneous and comprise a mixture of tumor and normal cells (endothelial cells, leukocytes, fibroblasts). For this reason, the copy number values corresponding to DNA alterations of tumor cells are inevitably "diluted" by the diploid values coming from normal cells. Differently, primary cultures were more homogeneous in terms of tumor component, as shown by their phenotypic characterization, and thus presented better defined CNAs. Tumor tissue heterogeneity reflects also on $\mathrm{LOH}$ detection. In fact, the software used in the analysis not only detected the allele retained by the tumor, but also the second allele still present in normal cells; in these cases the $\mathrm{LOH}$ call will be missed [33]. In our ccRCC primary cultures, instead, hemizygous deletions were accompanied by LOH calls, confirming the great sample homogeneity that enables to discriminate CNAs and $\mathrm{LOH}$. Thus, the presence of $\mathrm{LOH}$ calls in deleted regions could be adopted as a parameter to evaluate the degree of tumor sample purity and the level of normal cell contamination and consequently the origin of cultured cells, highlighting the power of SNP array technology with respect to CGH technique. Such an evidence appears more important taking into account that since today there is not a single universally applied method able to certainly make these evaluations [34].

The high cell homogeneity observed in our ccRCC cultures might explain also the additional CNAs found in 4 of them but not in original tissues. The acquisition of these alterations de novo during in vitro growth is unlikely since cultures were analyzed at first confluence. Moreover, the genomic analysis of some cultures at second and third confluences provided evidence that these cultures did not accumulate further alterations during passages and remained stable for at least 3 weeks. We could therefore conclude that these alterations might fail to be seen in the heterogeneous tumor tissues because present in a very small number of cells $(<20 \%)$, thus resulting undetectable for $\mathrm{CN}$ analysis algorithms $[11,24]$.

Actually, the true picture of genomic alterations occurring in a tumor can be obtained only performing a genomic analysis directly on tumor cells isolated, for example, by laser capture microdissection, which allows a $>90 \%$ of purity [35] and circumvents the problem of DNA alteration "dilution" due to tissue heterogeneity. However, such a useful technical approach has the not negligible inconvenience of providing cellular material not exploitable for eventual subsequent functional studies.

\section{Conclusions}

By performing the genome-wide copy number profiling of a collection of ccRCC primary cultures and corresponding tumor tissues, we demonstrated that these well-characterized primary cultures maintained the genomic alterations of parental tumors. Moreover, their DNA profile remained stable for at least 3 weeks, till to third confluence. Importantly, RCC primary cultures provided greater cell homogeneity and enrichment in tumor component than parental tissues, as proved also by phenotypic characterization, thus enabling a better discrimination of DNA alterations. In this context, SNP array technology demonstrated to be a powerful tool able to confirm the origin of cultured cells and to evaluate sample homogeneity and normal cell contamination. The observation that ccRCC primary cultures retain not only the phenotypic features and the proteomic profile of original tumor tissues but also their genomic profile proves that these short-term cultures are a reliable in vitro model that well represents ccRCC genetics and biology and that prospectively could be used for functional approaches.

\section{Additional material}

Additional file 1: Whole-genome maps of CN alterations and $\mathrm{LOH}$ events in the nine ccRCC primary cultures, using CNAG v3.0

software. Chromosomes are represented from $p$ to $q$ end (from left to right), with cytobands (black and white blocks), centromeres (green blocks) and heterocromatic regions (red and blue blocks). (a) Starting from the HMM-CN state data, regions of CN gain (upper red traces) and CN loss (lower green traces) are represented along each chromosome (from1 to 22). (b) Regions of statistically significant $\mathrm{LOH}$ (with $\mathrm{LOH}$ likelihood higher than 30) are represented with red traces along each chromosome. Chromosome $X$ was excluded from analyses. 
Additional file 2: Whole-genome view of copy number profile in $80 \mathrm{MLa}$ primary culture at first ( $\mathrm{p} 1)$ and second (p2) confluences, and in corresponding tumor tissue, using CNAG v3.0 software. Analysis was performed using CNAG V3.0 software, comparing primary culture at each passage and parental tumor tissue to the autologous blood sample. Chromosomes are represented horizontally, from 1 to 22 in different colors, separated by vertical bars. For each sample, the three tracks represent (on log scale): a) "copy number plot": copy number log ratio values of single SNPs; b) "copy number average": copy number log ratio values locally averaged on 10 contiguous SNPs; c) "allele-based analysis": copy number log ratio values for each allele (red and green lines).

Additional file 3: Copy number and LOH likelihood values for selected deleted regions in primary cultures and corresponding tissues, as calculated by CNAG v3.0 software. Starting from the Hidden Markov Model (HMM) copy number log2ratios exported for each SNP by CNAG V3.0 software, we calculated the mean CN log ratio value for each region (start and end positions are reported), both in primary cultures and parental tumor tissues. Also, mean LOH likelihood values were calculated for primary cultures and corresponding tissues. In the "CNAG detection" column, we define "CN loss signed" those deletions reaching software threshold to be visualized in the color-coded HMM-CN state track; similarly, "LOH signed" are those events considered as statistically significant by CNAG (with LOH likelihood higher than 30) and thus visualized in the color-coded HMM-LOH track.

Additional file 4: Partek Genomics Suite analysis: CN loss detection in 66SML primary culture and parental tissue by applying the two different algorithms HMM (Hidden Markov Model, the same used by CNAG software) and GS (Genomic Segmentation). Analysis was performed starting from CEL intensity files produced by Affymetrix GCOS software, and comparing each $\mathrm{CCRCC}$ primary culture and parental tissue to its autologous blood sample. Two different algorithms were used: HMM (Hidden Markov Model) and GS (Genomic Segmentation). Here we reported an example of the different output returned by the two algorithms for 66SML case. On the five chromosomes here displayed (chrs 1p, 2q, 3p, 9, 14q), the CN loss regions, even if clearly visible in the log ratio $\mathrm{CN}$ track (upper graph, in log2 scale), failed to be signed by the HMM algorithm in the tissue sample (middle track), exactly as observed in CNAG analysis. Differently, the GS algorithm was able to retrieve all these regions in tissue sample, visualizing them as green bars (bottom track).

\begin{abstract}
Acknowledgements
A thank to Dr. Paola Brambilla (Institute of Experimental Neurology, Scientific Institute of San Raffaele, Milan), Dr. Paolo Mocarelli (Desio Hospital, University of Milano-Bicocca), Dr. Silvano Bosari (Dept. Medicine, Surgery and Dentistry, Ospedale Maggiore Policlinico, University of Milan), Dr. Valentina Usuelli (Dept. Specialistic Surgical Sciences, Urology Unit, Ospedale Maggiore Policlinico, University of Milan). A special thank to Danila Coradini for scientific editing of the manuscript. This work was supported by grants from the Italian Ministry of University and Research: FIRB 2003 (n.

RBLA03ER38_004), PRIN 2006 (n. 69373), FIRB 2007 (Rete nazionale per lo studio del proteoma umano, n. RBRNO7BMCT_011).
\end{abstract}

\section{Author details}

'Institute for Biomedical Technologies (ITB), National Research Council (CNR), via Fratelli Cervi 93, 20090 Segrate, Milan, Italy. ${ }^{2}$ Department of Experimental Medicine, University of Milano-Bicocca, via Cadore 48, 20052 Monza, Milan, Italy. ${ }^{3}$ Department of Medicine, Surgery and Dentistry, Pathological Anatomy Unit, University of Milan, Ospedale Maggiore Policlinico, via Sforza 35, 20122 Milan, Italy. ${ }^{4}$ Department of Laboratory Medicine, Desio Hospital, University of Milano-Bicocca, via Mazzini 1, 20033 Desio, Milan, Italy. ${ }^{5}$ Dipartimento di Scienze e Tecnologie Biomediche, Università degli Studi di Milano, via Fratelli Cervi 93, 20090 Segrate, Milan, Italy.

\section{Authors' contributions}

IC carried out microarray experiments and bioinformatic analyses. EM and FF performed statistical and bioinformatic analyses. CB, SB, VDS and MAZ prepared primary cultures and performed phenotypic characterizations. EF and SF carried out histological classification of clinical cases and prepared DNA from tissue samples. SS prepared DNA from blood samples. FM participated in the study design. CB and RAP coordinated and supervised the study and, together with $I C$ and $C B$, wrote the manuscript. All authors read and approved the final manuscript.

\section{Competing interests}

The authors declare that they have no competing interests.

Received: 27 October 2010 Accepted: 13 June 2011

Published: 13 June 2011

\section{References}

1. Kovacs G, Akhtar M, Beckwith BJ, Bugert P, Cooper CS, Delahunt B, Eble JN, Fleming S, Ljungberg B, Medeiros L, Moch H, Reuter VE, Ritz E, Roos G, Schmidt D, Srigley JR, Storkel S, van den Berg E, Zbar B: The Heidelberg classification of renal cell tumours. J Pathol 1997, 183(2):131-133.

2. Hoglund M, Gisselsson D, Soller M, Hansen GB, Elfving P, Mitelman F: Dissecting karyotypic patterns in renal cell carcinoma: an analysis of the accumulated cytogenetic data. Cancer Genet Cytogenet 2004, 153(1):1-9.

3. Klatte T, Rao PN, de Martino M, LaRochelle J, Shuch B, Zomorodian N, Said J, Kabbinavar FF, Belldegrun AS, Pantuck AJ: Cytogenetic profile predicts prognosis of patients with clear cell renal cell carcinoma. J Clin Oncol 2009, 27(5):746-753.

4. Gunawan B, Huber W, Holtrup M, von Heydebreck A, Efferth T, Poustka A, Ringert RH, Jakse G, Fuzesi L: Prognostic impacts of cytogenetic findings in clear cell renal cell carcinoma: gain of 5q31-qter predicts a distinct clinical phenotype with favorable prognosis. Cancer Res 2001, 61(21):7731-7738.

5. Perego RA, Corizzato M, Brambilla P, Ferrero S, Bianchi C, Fasoli E, Signorini S, Torsello B, Invernizzi L, Bombelli S, Angeloni V, Pitto M, Battaglia C, Proserpio V, Magni F, Galasso G, Mocarelli P: Concentration and microsatellite status of plasma DNA for monitoring patients with renal carcinoma. Eur J Cancer 2008, 44(7):1039-1047.

6. Beroukhim R, Brunet JP, Di Napoli A, Mertz KD, Seeley A, Pires MM, Linhart D, Worrell RA, Moch H, Rubin MA, Sellers WR, Meyerson M, Linehan WM, Kaelin WG Jr, Signoretti S: Patterns of gene expression and copy-number alterations in von-hippel lindau disease-associated and sporadic clear cell carcinoma of the kidney. Cancer Res 2009, 69(11):4674-4681.

7. Young AC, Craven RA, Cohen D, Taylor C, Booth C, Harnden P, Cairns DA, Astuti D, Gregory W, Maher ER, Knowles MA, Joyce A, Selby PJ, Banks RE: Analysis of VHL Gene Alterations and their Relationship to Clinical Parameters in Sporadic Conventional Renal Cell Carcinoma. Clin Cancer Res 2009, 15(24):7582-7592.

8. Gordan JD, Lal P, Dondeti VR, Letrero R, Parekh KN, Oquendo CE, Greenberg RA, Flaherty KT, Rathmell WK, Keith B, Simon MC, Nathanson KL: HIF-alpha effects on c-Myc distinguish two subtypes of sporadic VHLdeficient clear cell renal carcinoma. Cancer Cell 2008, 14(6):435-446.

9. Dutt A, Beroukhim R: Single nucleotide polymorphism array analysis of cancer. Curr Opin Oncol 2007, 19(1):43-49.

10. Bacolod MD, Schemmann GS, Giardina SF, Paty P, Notterman DA, Barany F: Emerging paradigms in cancer genetics: some important findings from high-density single nucleotide polymorphism array studies. Cancer Res 2009, 69(3):723-727.

11. Goransson $H$, Edlund $K$, Rydaker M, Rasmussen $M$, Winquist J, Ekman $S$, Bergqvist M, Thomas A, Lambe M, Rosenquist R, Holmberg L, Micke P, Botling J, Isaksson A: Quantification of normal cell fraction and copy number neutral LOH in clinical lung cancer samples using SNP array data. PLoS One 2009, 4(6):e6057.

12. Balmain A, Gray J, Ponder B: The genetics and genomics of cancer. Nat Genet 2003, 33(Suppl):238-244.

13. Cifola I, Spinelli R, Beltrame L, Peano C, Fasoli E, Ferrero S, Bosari S, Signorini S, Rocco F, Perego R, Proserpio V, Raimondo F, Mocarelli P, Battaglia C: Genome-wide screening of copy number alterations and LOH events in renal cell carcinomas and integration with gene expression profile. Mol Cancer 2008, 7:6.

14. Tan X, Zhai Y, Chang W, Hou J, He S, Lin L, Yu Y, Xu D, Xiao J, Ma L, Wang G, Cao T, Cao G: Global analysis of metastasis-associated gene 
expression in primary cultures from clinical specimens of clear-cell renalcell carcinoma. Int J Cancer 2008, 123(5):1080-1088.

15. Craven RA, Stanley AJ, Hanrahan S, Dods J, Unwin R, Totty N, Harnden P, Eardley I, Selby PJ, Banks RE: Proteomic analysis of primary cell lines identifies protein changes present in renal cell carcinoma. Proteomics 2006, 6(9):2853-2864.

16. Hagenkord JM, Monzon FA, Kash SF, Lilleberg S, Xie Q, Kant JA: Arraybased karyotyping for prognostic assessment in chronic lymphocytic leukemia: performance comparison of Affymetrix 10K2.0, 250K Nsp, and SNP6.0 arrays. J Mol Diagn 2010, 12(2):184-196.

17. Heinzman JM, Brower SL, Bush JE, Silverman JF: Ex vivo enrichment of malignant carcinoma cells in primary culture. Pathology 2007, 39(5):491-494.

18. Perego RA, Bianchi C, Corizzato M, Eroini B, Torsello B, Valsecchi C, Di Fonzo A, Cordani N, Favini P, Ferrero S, Pitto M, Sarto C, Magni F, Rocco F, Mocarelli P: Primary cell cultures arising from normal kidney and renal cell carcinoma retain the proteomic profile of corresponding tissues. J Proteome Res 2005, 4(5):1503-1510.

19. Bianchi C, Bombelli S, Raimondo F, Torsello B, Angeloni V, Ferrero S, Di Stefano V, Chinello C, Cifola I, Invernizzi L, Brambilla P, Magni F, Pitto M, Zanetti G, Mocarelli P, Perego RA: Primary cell cultures from human renal cortex and renal-cell carcinoma evidence a differential expression of two spliced isoforms of Annexin A3. Am J Pathol 2010, 176(4):1660-1670.

20. Lin WM, Baker AC, Beroukhim R, Winckler W, Feng W, Marmion JM, Laine E, Greulich H, Tseng H, Gates C, Hodi FS, Dranoff G, Sellers WR, Thomas RK, Meyerson M, Golub TR, Dummer R, Herlyn M, Getz G, Garraway LA: Modeling genomic diversity and tumor dependency in malignant melanoma. Cancer Res 2008, 68(3):664-673.

21. Volchenboum SL, Li C, Li S, Attiyeh EF, Reynolds CP, Maris JM, Look AT, George RE: Comparison of primary neuroblastoma tumors and derivative early-passage cell lines using genome-wide single nucleotide polymorphism array analysis. Cancer Res 2009, 69(10):4143-4149.

22. De Witt Hamer PC, Van Tilborg AA, Eijk PP, Sminia P, Troost D, Van Noorden CJ, Ylstra B, Leenstra S: The genomic profile of human malignant glioma is altered early in primary cell culture and preserved in spheroids. Oncogene 2008, 27(14):2091-2096.

23. Sanjmyatav J, Schubert J, Junker K: Comparative study of renal cell carcinoma by CGH, multicolor-FISH and conventional cytogenic banding analysis. Oncol Rep 2005, 14(5):1183-1187.

24. Yamamoto G, Nannya Y, Kato M, Sanada M, Levine RL, Kawamata N, Hangaishi A, Kurokawa M, Chiba S, Gilliland DG, Koeffler HP, Ogawa S: Highly sensitive method for genomewide detection of allelic composition in nonpaired, primary tumor specimens by use of affymetrix single-nucleotide-polymorphism genotyping microarrays. Am J Hum Genet 2007, 81(1):114-126.

25. Young AN, Amin MB, Moreno CS, Lim SD, Cohen C, Petros JA, Marshall FF, Neish AS: Expression profiling of renal epithelial neoplasms: a method for tumor classification and discovery of diagnostic molecular markers. Am J Pathol 2001, 158(5):1639-1651.

26. Li G, Cuilleron M, Cottier M, Gentil-Perret A, Lambert C, Genin C, Tostain J: The use of MN/CA9 gene expression in identifying malignant solid renal tumors. Eur Urol 2006, 49(2):401-405.

27. Patard JJ, Fergelot P, Karakiewicz PI, Klatte T, Trinh QD, Rioux-Leclercq N, Said JW, Belldegrun AS, Pantuck AJ: Low CAIX expression and absence of VHL gene mutation are associated with tumor aggressiveness and poor survival of clear cell renal cell carcinoma. Int J Cancer 2008, 123(2):395-400.

28. Li G, Passebosc-Faure K, Lambert C, Gentil-Perret A, Blanc F, Oosterwijk E, Mosnier JF, Genin C, Tostain J: The expression of G250/mn/CA9 antigen by flow cytometry: its possible implication for detection of micrometastatic renal cancer cells. Clin Cancer Res 2001, 7(1):89-92.

29. Signoretti S, Regan M, Atkins M: Carbonic anhydrase IX as a predictive biomarker of response to kidney cancer therapy. BJU Int 2008, 101(Suppl 4):31-35.

30. Kovacs G, Szucs S, De Riese W, Baumgartel H: Specific chromosome aberration in human renal cell carcinoma. Int J Cancer 1987, 40(2):171-178.

31. Shi T, Dong F, Liou LS, Duan ZH, Novick AC, DiDonato JA: Differential protein profiling in renal-cell carcinoma. Mol Carcinog 2004, 40(1):47-61.

32. Bozic I, Antal T, Ohtsuki H, Carter H, Kim D, Chen S, Karchin R, Kinzler KW, Vogelstein B, Nowak MA: Accumulation of driver and passenger mutations during tumor progression. Proc Natl Acad Sci USA 2010, 107(43):18545-18550.

33. Lo KC, Bailey D, Burkhardt T, Gardina P, Turpaz Y, Cowell JK: Comprehensive analysis of loss of heterozygosity events in glioblastoma using the 100K SNP mapping arrays and comparison with copy number abnormalities defined by BAC array comparative genomic hybridization. Genes Chromosomes Cancer 2008, 47(3):221-237.

34. Ochs RL, Fensterer J, Ohori NP, Wells A, Gabrin M, George LD, Kornblith P: Evidence for the isolation, growth, and characterization of malignant cells in primary cultures of human tumors. In Vitro Cell Dev Biol Anim 2003, 39(1-2):63-70.

35. Nishidate T, Katagiri T, Lin ML, Mano Y, Miki Y, Kasumi F, Yoshimoto M, Tsunoda T, Hirata K, Nakamura Y: Genome-wide gene-expression profiles of breast-cancer cells purified with laser microbeam microdissection: identification of genes associated with progression and metastasis. Int J Oncol 2004, 25(4):797-819.

\section{Pre-publication history}

The pre-publication history for this paper can be accessed here: http://www.biomedcentral.com/1471-2407/11/244/prepub

doi:10.1186/1471-2407-11-244

Cite this article as: Cifola et al:: Renal cell carcinoma primary cultures maintain genomic and phenotypic profile of parental tumor tissues. BMC Cancer 2011 11:244.

\section{Submit your next manuscript to BioMed Central and take full advantage of:}

- Convenient online submission

- Thorough peer review

- No space constraints or color figure charges

- Immediate publication on acceptance

- Inclusion in PubMed, CAS, Scopus and Google Scholar

- Research which is freely available for redistribution

Submit your manuscript at www.biomedcentral.com/submit
C Biomed Central 